\title{
Experimental study of coherent betatron resonances with a Paul trap
}

\author{
S. Ohtsubo, M. Fujioka, H. Higaki, K. Ito, H. Okamoto, and H. Sugimoto \\ Graduate School of Advanced Sciences of Matter, Hiroshima University, 1-3-1 Kagamiyama, Higashi-Hiroshima 739-8530, Japan
}

\author{
S. M. Lund
}

Lawrence Livermore National Laboratory, Livermore, California 94550, USA

(Received 3 November 2008; revised manuscript received 2 February 2010; published 19 April 2010)

\begin{abstract}
Linear and nonlinear resonant instabilities of charged-particle beams traveling in periodic quadrupole focusing channels are studied experimentally with a compact non-neutral plasma trap. The present experiments are based on the idea that the collective motion of a beam in an accelerator is physically similar to that of a one-component plasma in a trap. A linear Paul trap system named "S-POD" (simulator for particle orbit dynamics) was developed to explore a variety of space-charge-induced phenomena. To emulate lattice-dependent effects, periodic perturbations are applied to quadrupole electrodes, which gives rise to additional resonance stop bands that shift depending on the plasma density. It is confirmed that an $m$ th-order resonance takes place when the corresponding tune of an $m$ th-order collective mode $\Omega_{m}$ is close to a half integer.
\end{abstract}

DOI: 10.1103/PhysRevSTAB.13.044201

PACS numbers: 29.27.Bd, 41.85.Ja, 52.27.Jt

\section{INTRODUCTION}

The quality of a charged-particle beam can be seriously deteriorated by collective instabilities originating from space-charge self-fields [1-3]. Recent interest in highpower machines that transport large numbers of hadrons has made it more important to understand this type of collective phenomena [4,5]. Progress in beam cooling techniques has also motivated such studies because Coulomb interactions among particles are strengthened at low temperature $[6,7]$.

In order to conduct systematic investigations of spacecharge-dominated beam dynamics, we developed a tabletop experimental tool called "S-POD" (simulator for particle orbit dynamics) [8,9]. The main components of S-POD include a radio-frequency quadrupole trap (linear Paul trap) [10,11], various DC and AC power supplies, a vacuum system, a laser cooler, an electron gun to ionize neutral gas, several diagnostics, and a comprehensive computer control system. Although Paul ion traps have been widely utilized for diverse purposes associated with frequency standards, Coulomb crystals, quantum computing, high-accuracy spectroscopy, and others [12-17], we designed and constructed S-POD solely for experimental beam physics. Our linear Paul trap consists of several, electrically isolated quarupoles configured to allow us to condition the plasma (e.g. ion species selection, plasma stacking, noise reduction, etc.) [9]. A similar system has been built at Princeton Plasma Physics Laboratory and is being employed by Gilson and co-workers to explore various fundamental beam dynamics issues [18-21].

The idea of using a compact non-neutral plasma trap for experimental beam physics was first proposed by Okamoto and Tanaka [22]. Trap experiments clearly have many advantages compared to conventional approaches relying on actual accelerators and beam transport systems. For instance, a typical trap system is tabletop size and can explore long-length issues far cheaper than constructing a long accelerator. It is possible to vary tunes and particle densities, over a wide range of values. Since the plasma motion is nonrelativistic, radioactivation due to particle losses during beam instability experiments is not an issue. The plasma centroid is at rest in the laboratory frame, which enables us to observe the collective behavior of the plasma at high resolution and precision. The S-POD experiment is thus useful to clarify the basic mechanisms of various space-charge effects.

In this paper, we report on recent S-POD experiments performed to identify the parameter regions in which an intense hadron beam becomes unstable in a periodic quadrupole lattice due to collective resonances. Periodic perturbations are intentionally added to the focusing quadrupole field in order to study the dependence of stop bands on focusing properties. A family of linear and nonlinear resonances are excited by varying the periodicity and amplitude of applied focusing forces. Although mechanical errors of the trap somewhat enhance nonlinear resonances, such instability can occur in an intense beam even if the driving force is perfectly linear [23,24]. In Sec. II, we outline the mechanism of coherent resonances including space-charge effects. A brief overview is given in Sec. III of the operational modes of S-POD. The magnitudes of possible nonlinear components in the applied focusing field are estimated employing a three-dimensional (3D) Maxwell equation solver. Section IV is devoted to showing experimental results obtained with S-POD. In Sec. V, we compare the experimental observations with particle-incell (PIC) simulation results and confirm good qualitative agreement as to resonance locations. Concluding remarks are made in Sec. VI. 


\section{COHERENT RESONANCES}

It is well known that the amplitude of the betatron oscillation of a particle traveling in an accelerator grows due to resonant interaction with the external beam focusing force. The periodic nature of the machine lattice is responsible for this instability; in fact, no resonance occurs in a uniform focusing channel [25]. In a single-particle point of view, it is straightforward to analyze this instability mathematically $[26,27]$. The self-consistent analysis of resonances, however, becomes extremely difficult once the Coulomb interactions among particles are taken into account. Provided that the multiparticle system of our interest is governed by the Hamiltonian $H$ and collisional effects are negligible, the phase-space distribution function $f$ obeys the Vlasov equation,

$$
\frac{\partial f}{\partial s}+[f, H]=0,
$$

where the independent variable $s$ is the path length along the design beam orbit, and [,] stands for the Poisson bracket. The task is to determine the $s$ evolution of $f$ from the initial form of $f$.

Let us now ignore the longitudinal $(z)$ degree of freedom for an unbunched $(\partial / \partial z=0)$ beam because we are particularly interested in transverse ( $x$ - and $y$-plane) resonances. Then, the Hamiltonian of a particle evolving in the alternating-gradient (AG) focusing fields of a transport lattice can be expressed as

$$
H=\frac{p_{x}^{2}+p_{y}^{2}}{2}+\frac{1}{2} K(s)\left(x^{2}-y^{2}\right)+\frac{q \phi}{m_{0} \gamma^{3}(\beta c)^{2}} .
$$

Here, $m_{0}$ and $q$ denote the rest mass and charge state of the particle, $\beta=$ const and $\gamma=1 / \sqrt{1-\beta^{2}}$ are the usual axial Lorentz factors, $c$ is the speed of light in vacuum, and the function $K(s)$ depends on the geometry and excitation of focusing optics making up the transport lattice of the accelerator. The electrostatic potential $\phi$ satisfies the transverse Poisson equation,

$$
\left(\frac{\partial^{2}}{\partial x^{2}}+\frac{\partial^{2}}{\partial y^{2}}\right) \phi=-\frac{q}{\varepsilon_{0}} \iint f d p_{x} d p_{y} .
$$

Hofmann et al. treated this set of closed equations selfconsistently without employing the smooth approximation and numerically found the stop bands of coherent resonances for a Kapchinsky-Vladimirsky (KV) beam with a matched envelope [23]. Before this seminal work, Gluckstern analytically derived the collective modes of a $\mathrm{KV}$ beam propagating in a continuous focusing channel [28].

A successful attempt to solve the Vlasov-Poisson equations for a hadron beam with strong space charge was made by Sacherer over 40 years ago [3]. His theory is one dimensional and applies to a uniform-density beam but is purely analytic. The instability criterion concluded by
Sacherer from the full Vlasov dispersion relation is often referred to as the coherent resonance condition $[3,29]$ :

$$
\Omega_{m} \approx n,
$$

where $m$ and $n$ are both positive integers, and $\Omega_{m}$ represents the tune of the $m$ th-order coherent mode. Note, however, that each collective resonance forms an instability band whose width becomes wider as the beam density increases.

Any real beam consists of many charged particles and is best regarded as a collective object rather than the collection of single particles. The beam motion as a whole can be expressed as a superposition of various collective modes whose tunes are different and depend on the beam density. Equation (4) indicates that the beam becomes unstable when one of the coherent tunes is near an integer. According to Sacherer $\Omega_{m}=m\left(\nu_{0}-C_{m} \Delta \nu\right)$, where $\nu_{0}$ is the bare betatron tune of the machine, $\Delta \nu$ is the tune shift induced by space charge (often called incoherent tune shift), and $C_{m}$ is a constant parameter smaller than unity. Substitution of this relation into Eq. (4) yields [30]

$$
\nu_{0}-C_{m} \Delta \nu \approx \frac{n}{m} .
$$

An important fact is that the left-hand side is different from the effective incoherent tune $\nu_{0}-\Delta \nu$. Since $C_{m}<1$ for all modes $(m>1)$, the maximum tune shift to avoid resonance crossing can be somewhat larger than the limit obtained from the incoherent condition $\nu_{0}-\Delta \nu \approx n / m$; this fact changes the theoretical estimate for the peak current that can be stored in circular machines.

It was pointed out, recently, that coherent resonances also take place under the condition [24]

$$
\Omega_{m} \approx \frac{n}{2}
$$

which includes Eq. (4) as a special case. This leads to

$$
\nu_{0}-C_{m} \Delta \nu \approx \frac{n}{2 m} .
$$

Equation (7) shows that the linear resonance $(m=2)$ occurs when the tune is near a quarter integer (not a half integer). The strength of each resonance depends on the lattice design, beam density, and the magnitudes of error fields; all resonance stop bands predicted by Eq. (7) are not necessarily visible in actual experiments. Deviations of the distribution from the matched KV form can wash out instabilities and Landau damping can also suppress instabilities. In any case, there exist twice as many resonance stop bands as predicted by Eq. (5). So-called imperfection fields from lattice errors do not essentially affect this criterion while they make the resonances of corresponding orders much stronger. Even if the lattice contains no errors and thus the driving force is completely linear, all modes with $m>2$ can be unstable due to space-charge nonlinearity [24]. 


\section{S-POD}

\section{A. Principle}

In the center-of-mass frame (say, the $s$ domain), a beam can be regarded as an ensemble of many identical particles focused by a linear external force. Not surprisingly, such a dynamical system can be represented in the laboratory frame (the time domain) with a one-component plasma trap [22]. Since the collective Coulomb potential of a nonneutral plasma in a trap satisfies the Poisson equation and the phase-space distribution approximately obeys the Vlasov equation, the correspondence between the $s$ and time domains is given solely by the Hamiltonians. If the plasma Hamiltonian takes the same form as the beam Hamiltonian in Eq. (2), the two systems can be considered physically equivalent. Consider an infinitely long plasma column transversely confined by a radio-frequency (rf) quadrupole potential. The Hamiltonian describing the motion of individual particles when the axial motion is neglected is given by

$$
H_{\mathrm{P}}=\frac{p_{x}^{2}+p_{y}^{2}}{2}+\frac{1}{2} K_{\mathrm{rf}}(t)\left(x^{2}-y^{2}\right)+\frac{q \phi}{m_{0} c^{2}},
$$

where $K_{\mathrm{rf}}(t)$ is a periodic function proportional to the $\mathrm{rf}$ waveform of the quadrupole potential. It is now evident that a Paul trap can be employed to experimentally simulate the beam motion governed by the Hamiltonian (2).

Equation (8) suggests that S-POD experiments at present are limited to the study of collective effects in which momentum dispersion does not play a substantial role. Such a very compact experimental facility can, nevertheless, provide useful information on many important subjects associated with high-power and/or low-emittance beams.

\section{B. Experimental apparatus}

As sketched in Fig. 1, each quadrupole rod of our Paul trap has been axially divided into three pieces, which allows a variety of experiments and plasma manipulation

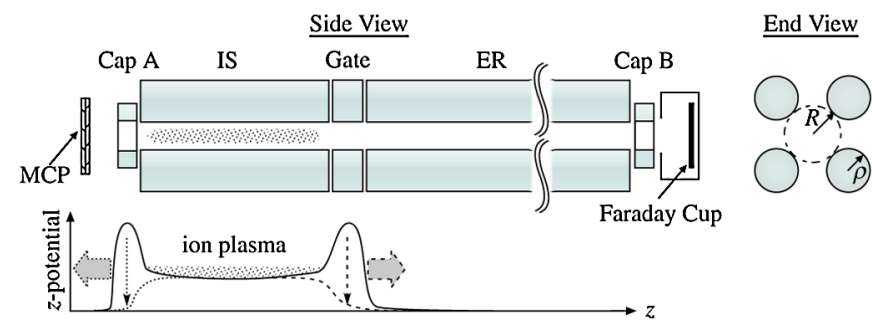

FIG. 1. (Color) S-POD setup for resonance experiments. An ion plasma is generated and confined in the ion source (IS) region using electron impact ionization of a neutral gas. After shutting down the electron beam for ionization and evolving the plasma for a limited number of rf focusing periods, the plasma is extracted for measurements by dropping the bias potential on either the gate or end cap A.
[9]. The three sections, i.e., ion source (IS), gate, and experiment region (ER), are electrically isolated and can be independently excited with AC and DC power sources. It is possible to configure DC biases to form two separate axial potential wells in the ER and the IS. We can even accumulate plasmas in the ER by controlling the bias voltages on the three sections and transporting ions from the IS [9]. The axial lengths of the IS, gate, and ER sections are 50, 9, and $100 \mathrm{~mm}$, respectively; the S-POD experiment is much more compact than the dedicated Paul trap simulator experiment at Princeton which is $2.8 \mathrm{~m}$ long [19]. Since our system is compact and inexpensive, we have constructed several Paul traps that are optimized for different experiments. The multisection Paul trap employed here is one of them.

Non-neutral plasmas are generated in the IS with a lowenergy electron beam that ionizes neutral gas forming primarily charge-state one $\mathrm{Ar}^{+}$ions (typically, 99\% pure). Other charge states are lost during the long plasma accumulation cycle (typically, around $10^{6}$ focusing rf periods). We can further improve the purity, if necessary, by applying bias voltages to the quadrupole electrodes, which result in undesired $q / \mathrm{m}$ ratio particles being lost transversely. We can also employ other ion species $\left(\mathrm{N}^{+}, \mathrm{Ca}^{+}\right.$, etc.). All three sections are excited with rf potentials of quadrupole symmetry to confine the $\mathrm{Ar}^{+}$ion plasma transversely both during transport experiments and plasma accumulation and extraction. For longitudinal confinement, we apply DC voltages on the end caps (cap A and cap B) or one of the three sections. DC biases can be added to the rf voltages in the IS and/or ER to accelerate ions toward the detectors at both ends of the trap (see Fig. 1). In the present experiments, cap A and the gate are biased to store ions in the IS region. After storage there for $\sim 1000 \mathrm{rf}$ periods, the plasma is transported through the gate and the ER to the Faraday cup by switching the DC bias on the gate. On the other side of the trap (to the left of cap A), a microchannel plate (MCP) with a phosphor screen and a CCD camera are placed which enable us to measure the transverse density profile of the plasma. For the profile measurement, the bias on cap A instead of that on the gate is switched. The number of ions measured in the Faraday cup is noticeably reduced when the operating point of the trap is close to a resonance (see Sec. IV B 1). We have developed a programmable switching system to control the electron gun, $\mathrm{AC}$, and DC power supplies. The system automatically executes a sequence of desired plasma manipulations while storing measurement data in a personal computer.

The chamber is connected to a turbo molecular pump (450 liter/sec) that achieves a vacuum pressure of $10^{-8} \mathrm{~Pa}$. We can change the number of confined ions by introducing different amounts of Ar gas into the chamber. The maximum value of tune depression reached by this procedure is about 0.8 . In future experiments, we will use a laser cooler that can control the plasma temperature. This 
can allow us to achieve much lower values of tune depression. The typical lifetime of the ion plasma is a few seconds, corresponding to millions of rf periods. The plasma lifetime can be significantly improved by conditioning the plasma with a cooling system or by simply reducing the number of stored ions.

\section{Nonlinear applied field components}

Before proceeding to results of resonance experiments, for later convenience we estimate the strengths of nonlinear components in the plasma confinement force. Numerous coherent resonances can be excited even without imperfect (nonlinear) fields, due to nonlinear spacecharge fields. Resonances induced by space-charge nonlinearity can be enhanced by nonlinear applied fields.

Ideally, the transverse profile of the quadrupole electrodes should be parabolic in order to make the plasma confinement field exactly linear. In practice, most linear Paul traps, including ours, employ cylindrical rods to simplify fabrication at the expense of generating nonlinear components in the fields. Provided that the system has no mechanical alignment errors, every fourth multipole harmonic component $(m=2,6,10, \ldots)$ appears in the $2 \mathrm{D}$ transverse field. The lowest-order nonlinear term $(m=6)$ can be minimized by choosing $\rho / R \approx 1.15$, where $R$ is the minimum distance from the trap axis to the surface of the electrodes of radius $\rho[8,31]$. The strengths of the nonlinear field terms are then suppressed to well below $0.1 \%$ of the fundamental quadrupole term at the aperture extent $(r=$ $R$ ). Therefore, only very weak nonlinearities are present in the idealized geometry of our experiments. The actual Paul trap, however, contains mechanical alignment errors that can considerably amplify nonlinear components of all orders. In fact, we have observed particle losses due to low-order resonances associated with the $m=3,4$, and 5 multipole terms which are not present in the ideal geometry [32].

To make a rough estimate of error-induced nonlinearity, we use the software CST STUDIO [33] to analyze the fields generated by the $2 \mathrm{D}$ transverse geometry of our trap. $\rho$ and $R$ of our trap have been chosen to be 5.75 and $5 \mathrm{~mm}$, respectively, meeting the requirement $\rho / R \approx 1.15$. Figure 2 shows error fields produced both in the absence of alignment errors and when the quadrupole rods are displaced randomly from the ideal positions. The random displacements have a Gaussian distribution with the standard deviation of 0.05 or $0.1 \mathrm{~mm}$, which is consistent with likely root-mean-squared (rms) misalignments in our system. We find that the amplitude of the $m=3$ multipole field is finite and significantly increased to over $0.1 \%$ of the quadrupole field amplitude. The second lowest-order nonlinearity, the $m=4$ multipole field is also at a nonnegligible level. Other higher-order components are weaker, so we expect their effects to be less important. The individual rods can also be tilt misaligned relative to

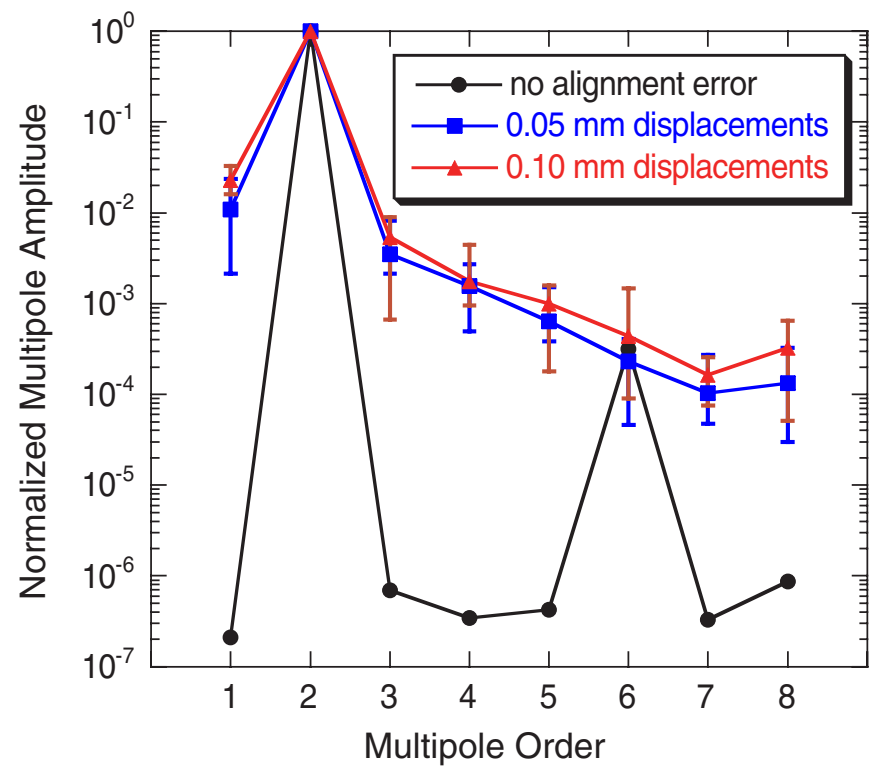

FIG. 2. (Color) Multipole amplitudes of nonlinear fields generated by nonideal electrode shape and mechanical alignment errors in the Paul trap. Rms transverse displacement errors of the electrode rods assumed in these examples are $0.05 \mathrm{~mm}$ (blue) and $0.1 \mathrm{~mm}$ (red). In this example, ten different sets of Gaussian random numbers are used to define the displacements of four rods. The strengths of multipole fields have been normalized by the quadrupole amplitude.

each other (overall tilt of system is irrelevant). One might expect this error source to introduce similar magnitude nonlinear fields as for the case of the transverse displacement misalignments analyzed.

The amount of a possible tune shift caused by transverse displacement misalignments of the electrodes was evaluated with CST STUDIO. A number of numerical trials with various random misalignments indicate that the amplitude of the linear focusing field is somewhat affected by the specific mechanical error (while its average over many trials stays almost constant). The possible maximum tune shift becomes larger as we increase the rms size of the misalignments. The shift, however, is less than $\pm 4 \%$ with an rms error of $0.1 \mathrm{~mm}$. Experimental observations shown in Sec. IV suggest that the error-induced shift of the bare tune is negligible in our case or, at least, much smaller than the space-charge-induced tune shift.

\section{Lattice-dependent driving forces}

The power supply system for S-POD is controlled with LABVIEW [34]. It is straightforward to produce complex periodically varying voltages that emulate a wide variety of $K_{\mathrm{rf}}(t)$ lattice functions allowing experiments for many different AG lattice structures. The essence of the coherent resonance mechanism can, however, be studied systematically with a simple sinusoidal waveform of rf. Little difference in resonance structure is observed for simple variations in the periodic form of $K_{\mathrm{rf}}(t)$. As discussed in 
(a) Perfect Lattice

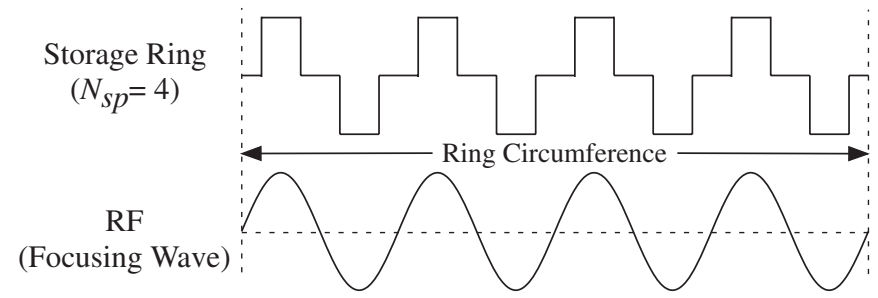

(b) With Imperfection

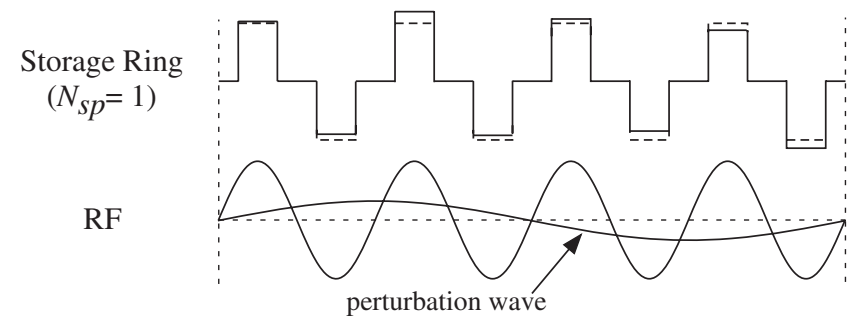

FIG. 3. Example of a ring lattice emulation scheme in the SPOD experiments. A single FODO period corresponds to a single sinusoidal rf period. Error fields reduce the strict lattice periodicity to unity, which can be approximately modeled by superimposing a perturbation wave (shown in exaggerated scale).

Sec. II, a resonance is excited when the corresponding coherent tune is close to either an integer or a half integer or, in other words, the frequency of a collective mode equals the frequency or half frequency of one of the many Fourier harmonics composing the periodic lattice function $K_{\mathrm{rf}}(t)$. For instance, consider a storage ring composed of four identical FODO periods and ignore any focusing effects of bending magnets which are not essential in the present study. The lattice superperiodicity of the ring $N_{s p}$ is four as shown in Fig. 3(a). In this perfect case with no field imperfections, the Fourier expansion of the one-turn driving field contains only every four harmonics, which is why a machine with higher lattice periodicity is generally preferred from a resonance point of view [35]. For perturbation with superperiod $N_{s p}$, the coherent resonance condition in Eq. (7) becomes

$$
\nu_{0}-C_{m} \Delta \nu \approx N_{s p} \frac{n}{2 m} .
$$

All real machines have lattice imperfections. However, the amplitudes of Fourier harmonics generated by weak imperfections are small. The growth rates of corresponding error-induced resonances should thus be small as long as the machine is carefully constructed.

According to the Vlasov theory by Hofmann et al. [23], the stop band of a matched KV distribution in a FODO quadrupole lattice with 50\% filling factor is almost identical to that of sinusoidal focusing. This is reasonable because the matched beam envelopes in both cases are similar for the same values of phase advance. We can, therefore, employ the simple waveform in the lower picture of Fig. 3(a) to analyze more typical FODO ring lattices. When the ring contains error fields, the strict superperiodicity turns to unity $\left(N_{s p}=1\right)$ as illustrated in Fig. 3(b). To study this situation approximately, we add a sinusoidal perturbation wave to the original harmonic focusing potential. The wavelength of the perturbation is equal to $N_{s p}$ times the single-cell length. We can add, if desired, many perturbation waves of different wavelengths either individually or in superposition.

\section{EXPERIMENTAL RESULTS}

We carry out a number of systematic resonance experiments with S-POD, changing the focusing periodicity $N_{s p}$ and other fundamental parameters. Since data obtained are analogous for other values of $N_{s p}$, we only describe results on $N_{s p}=12$. The rf frequency of the primary focusing field was chosen to be $996 \mathrm{kHz}$ throughout the experiments. The bare betatron tune per single turn around the ring $\nu_{0}$ can vary from 0 to $6(=0.5 \times 12)$ because the maximum phase advance achievable in a FODO period is 180 degrees before reaching the first single-particle stop band. The rf amplitude necessary for $\nu_{0}=6$ is $93 \mathrm{~V}$. The bare horizontal and vertical tunes, $\nu_{0 x}$ and $\nu_{0 y}$, satisfy $\nu_{0 x}=\nu_{0 y}$ in the Paul trap, and we denote $\nu_{0 x}=\nu_{0 y}=\nu_{0}$.

Figure 4 shows the rf waveforms employed to investigate the lattice dependence of resonant instability. Figure 4(a) corresponds to the perfect lattice with no errors over the 12 periods of the whole ring. The superperiod of an actual storage ring, however, often contains several unit FODO periods. To consider such common situations, we

(a) Primary Focusing Wave (12 FODO periods)

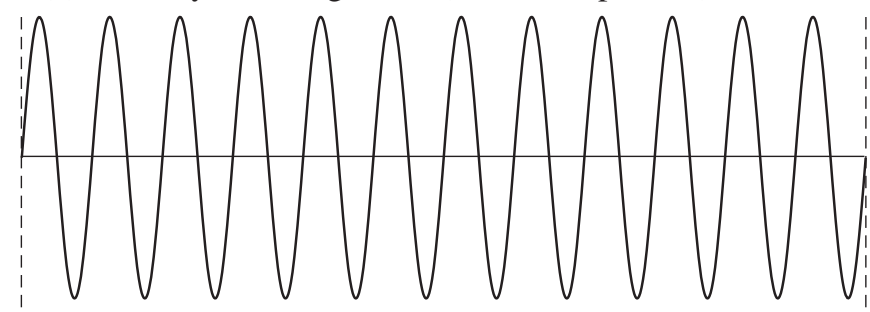

(b) Lattice-induced Wave $\left(N_{s p}=3\right)$

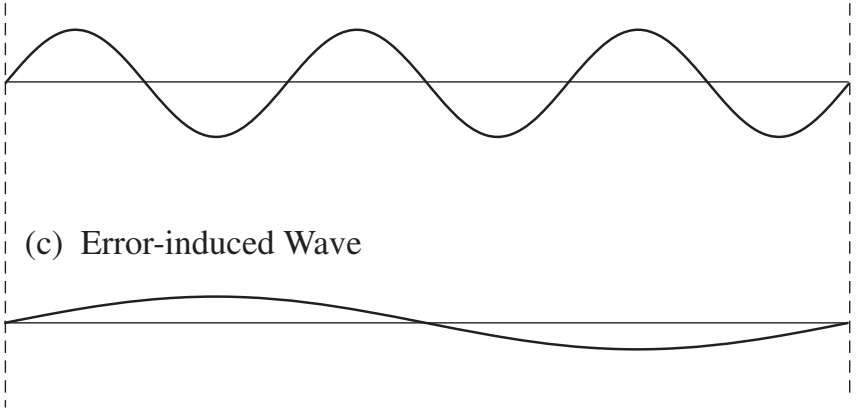

FIG. 4. Rf waveforms employed in the S-POD resonance experiments. 
superimpose an $\mathrm{rf}$ field whose wavelength is a proper integer multiple of the primary FODO wavelength. The case of Fig. 4(b) represents a ring composed of three superperiods, each of which contains four FODO periods [36]; the frequency of this lattice-induced wave is $249 \mathrm{kHz}$ $(=996 \mathrm{kHz} / 4)$. The effect of error fields is studied by further superimposing a perturbation wave of frequency $83 \mathrm{kHz}(=996 \mathrm{kHz} / 12)$ as shown in Fig. 4(c).

We use $\mathrm{Ar}^{+}$ions produced in the IS and confined them typically for $1 \mathrm{msec}$ corresponding to about 100 turns around the model ring. We have extended the storage time up to $10 \mathrm{msec}$, finding no essential change in the results except that resonant particle losses become more prominent. The plasma density is varied over a limited range by controlling the electron-beam current, ionization time, and the amount of gas. Our plasma accumulation procedure results in a typical transverse plasma extent of less than about $1.5 \mathrm{~mm}$ in radius. The long particle accumulation time is expected to result in a plasma that is well matched to the continuing rf potential. Since the IS region is $50 \mathrm{~mm}$ long, the ion cloud is axially extended like a long bunch [37]. The longitudinal plasma confinement potential is not parabolic but rather flat over the central region of the IS because the DC potentials on cap A and the gate are shielded by the long quadrupole rods in the IS. Each ion bounces back and forth along the axis without experiencing the longitudinal confinement force except for a small fraction of the bounce period when axially reflected near the ends. The longitudinal temperature of the plasma, which directly corresponds to the energy spread in the beam frame, is typically a few thousand kelvin (axial rms velocity $\sim 600 \mathrm{~m} / \mathrm{sec}$ ); it is, however, not essential to the fundamental features of transverse resonance.

\section{A. Low-density regime}

When the number of ions is relatively low, the plasma is approximately in an emittance-dominated state where the collective nature associated with the Coulomb force does not play a significant role. It should therefore be possible to predict the plasma behavior to a high degree of accuracy using a simple single-particle tracking code. We developed a 3D tracking code that can model the experimental procedure outlined above. The 3D rf field data necessary for the simulation is obtained in advance with CST STUDIO and imported into the code. The typical average mesh size of the imported field is $0.5 \mathrm{~mm}$, which is found to be sufficient according to Fourier analysis for a bare-tune error of less than $2.5 \%$. Although we launched many particles in each simulation to accurately sample an initial distribution, no Coulomb interactions were included. Example simulation results are given in Fig. 5 where two cases of ion losses as a function of $\nu_{0}$ both without $\left(N_{s p}=12\right)$ and with $\left(N_{s p}=3\right)$ perturbation are considered. The initial particle distribution employed here is spatially uniform in the transverse directions and chopped to the axial length of the trap. To

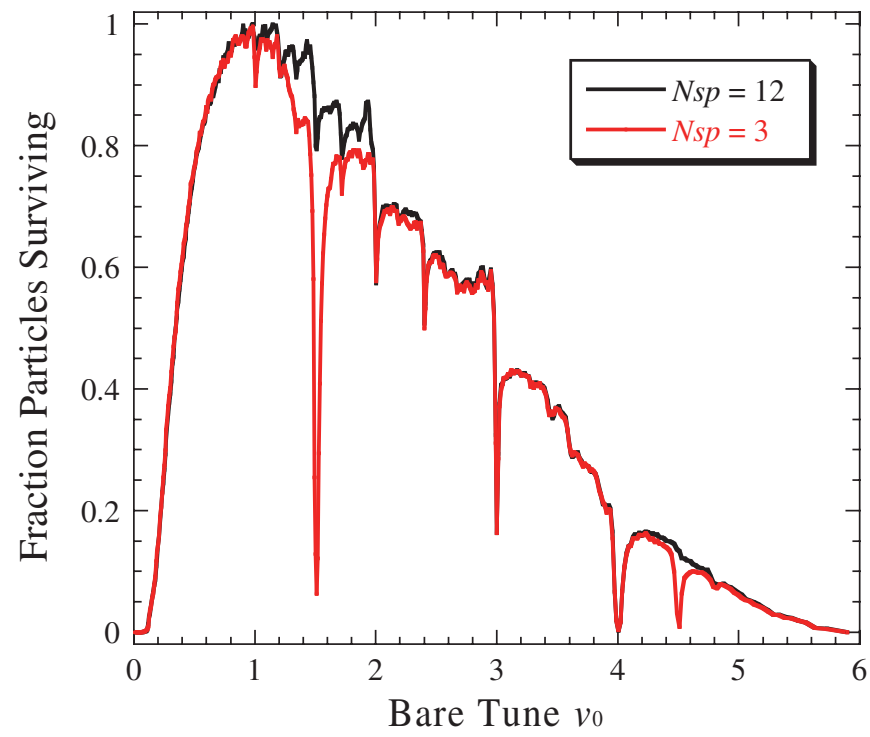

FIG. 5. (Color) Results of 3D tracking simulations without space charge. The number of Ar ions surviving after a $10 \mathrm{msec}$ storage time in the IS is plotted as a function of the one-turn bare betatron tune $\nu_{0}$. The ion number has been normalized with its peak value. The black and red curves show results for no perturbation $\left(N_{s p}=12\right.$, upper waveform in Fig. 4) and a threefold symmetric perturbation $\left(N_{s p}=3\right.$, middle waveform in Fig. 4) added to the primary wave. The amplitude of the perturbation is $0.3 \%$ of the primary wave. A representative sample of displacement misalignments of the quadrupole electrodes has been incorporated in the simulations.

clarify the stop band locations, the plasma storage time has been extended to $10 \mathrm{msec}$ in these simulations. Particles stored are measured by switching off the DC potential on the gate electrodes and counting particles streaming out of the trap (see Fig. 1). The black line corresponds to the 12fold symmetric ring while the red line to the threefold symmetric ring. Several sharp ion losses can be seen in both cases. The major instability occurring near $\nu_{0}=4$ is caused by a third-order resonance, which indicates that low-order error fields are not negligible as expected. Other sharp drops occurring at $\nu_{0} \approx 1.5,3.0$, and 4.5 are attributed primarily to linear resonances. The last one at $\nu_{0} \approx 4.5$ disappears when the threefold symmetric perturbation is switched off.

Experimental results corresponding to the simulation results in Fig. 5 are presented in Fig. 6. We employ the same measurement procedure and parameters as assumed in the numerical simulations. Since the Faraday cup does not have sufficient sensitivity at low ion current, we employed the MCP for low-density regime experiments. The results in Fig. 6 for the location of the resonance features are in good agreement with the tracking simulations in Fig. 5. However, the overall shape of the curves away from resonances varies significantly. These differences may be due to different initial distributions of particles, Coulomb collisions, scattering with neutral gasses, and multispecies effects. 


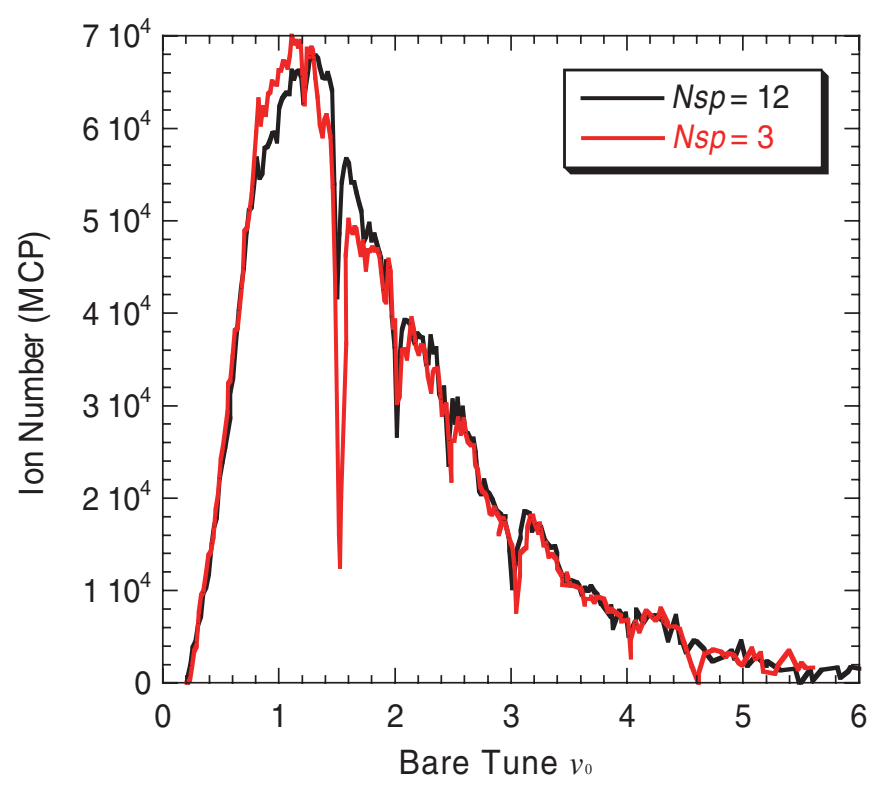

FIG. 6. (Color) Experimental results obtained under the same conditions as modeled in the tracking simulations in Fig. 5.

In these experiments, ions are produced by applying an electron beam to neutral atoms. The e-beam goes across the plasma confinement region transversely, ionizing the atoms everywhere within the transverse aperture near the axial center of the IS. The plasma is thus initially formed within the full acceptance of the trap. When the tune is near zero, the transverse focusing force is weak and, consequently, we lose many particles, since the transverse plasma size is too large to be confined within the aperture radius $R$. On the other hand, in the high-tune range, a large matched envelope oscillation associated with strong quadrupole focusing also leads to significant particle losses. At higher density, too strong focusing is dangerous also due to strong resonance instability. The betatron phase advance per single FODO period is generally chosen below 90 degrees (corresponding to $\nu_{0}=3$ in the present case) to suppress linear resonance problems. In the following studies of the high-density regime, we therefore concentrate on experimental data in the bare-tune range of $0<\nu_{0}<3.5$.

\section{B. High-density regime}

We carried out systematic experiments, confining 100 times more ions in the Paul trap. The stored ions were measured with the Faraday cup after passing through the ER. Particle losses during the transport process through the ER to the Faraday cup are negligible [9].

\section{Perfect lattice}

We temporarily, for simplicity, ignore the space-chargeinduced tune shift $\Delta \nu$. The resonance condition in Eq. (9) then gives $\nu_{0} \approx N_{s p} n / 2 m$. When $N_{s p}=3$, we may encounter linear coherent resonances at $\nu_{0} \approx 3 n / 4$, third- order resonances at $\nu_{0} \approx n / 2$, and fourth-order resonances at $\nu_{0} \approx 3 n / 8$. As previously discussed, some of these resonances can be enhanced depending on the form of external driving forces. We present typical experimental observations in Fig. 7 where the $N_{s p}=3$ wave has been added to the primary focusing wave of $N_{s p}=12$. The amplitude of the $N_{s p}=3$ wave was fixed at $0.1 \%, 0.5 \%$, and $1.0 \%$ of the $N_{s p}=12$ wave in each sequence of experiments. We find major stop bands, regardless of the perturbation strength, near $\nu_{0} \approx 2$ and 3 that are due mainly to third-order $(n=4)$ and second-order $(n=4)$ resonances, respectively. These two stop bands appear even when the perturbation wave is absent [38]. The slight shifts of the stop bands from the integers are attributed to the space-charged-induced detuning of the collectivemode frequencies. Because the Coulomb potential depresses the effective tune, the stop bands are shifted to the right (i.e., higher bare-tune values). Figure 8 shows the time evolution of the ion loss behavior observed at $\nu_{0} \approx$ 1.66 when the initial ion number is near $10^{7}$. Consistent with Fig. 7, strong instability-induced losses take place at this tune only when the $N_{s p}=3$ perturbation wave is introduced. Almost no ion losses occur without the perturbation $\left(N_{s p}=12\right)$, while a sharper drop of ion number is caused by larger a perturbation amplitude.

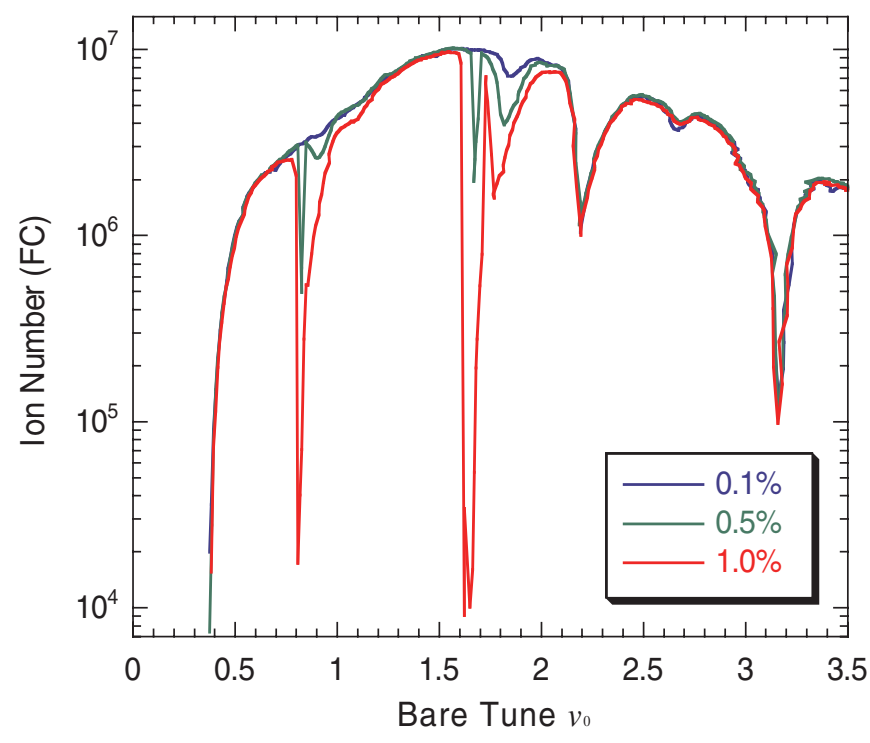

FIG. 7. (Color) Experimental results in the high-density regime where the number of trapped ions is about 100 times more than presented in Fig. 6. Three different amplitudes of the $N_{s p}=3$ wave have been considered; $0.1 \%, 0.5 \%$, and $1.0 \%$ of the $N_{s p}=$ 12 primary-wave amplitude. The instability region near $\nu_{0} \approx$ 0.75 contains two overlapping resonances that appear to correspond to mode numbers $(m, n)=(2,1)$ and $(4,2)$. Large particle losses near $\nu_{0} \approx 1.5$ are likely caused by the instability of linear and fourth-order collective modes with $(m, n)=(2,2)$ and $(4,4)$. The plasma storage time is $1 \mathrm{msec}$. 


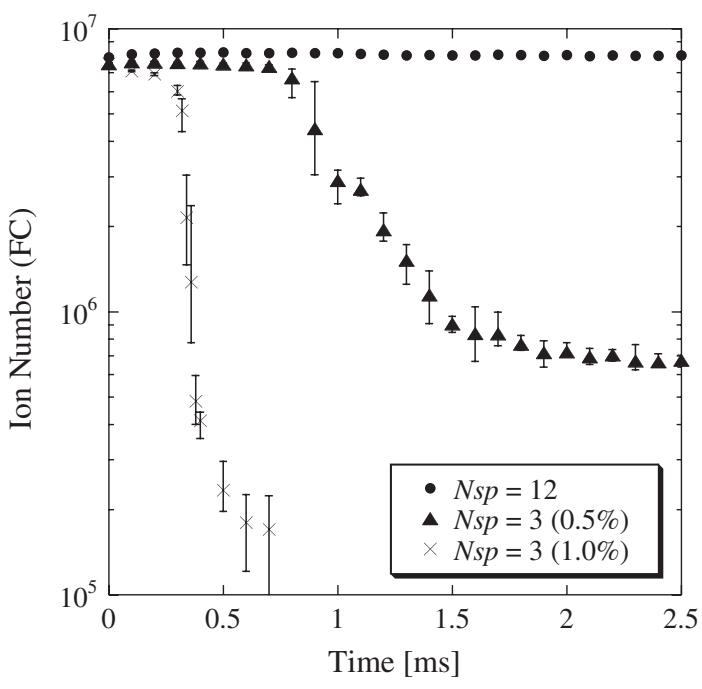

FIG. 8. Time evolution of the experimentally measured ion number confined in the trap. The amplitude of the primary rf field is fixed at $38.9 \mathrm{~V}$ corresponding to $\nu_{0} \approx 1.66$. Circles $(\mathbf{O})$ correspond to measurements of the ideal 12-fold symmetric $\left(N_{s p}=12\right)$ case where no perturbation is present. Crosses $(\times)$ and triangles $(\boldsymbol{\Delta})$ represent measurements when the perturbation waves are added to the $N_{s p}=12$ primary focusing wave. The amplitude of the $N_{s p}=3$ perturbation wave is given relative to the primary-wave amplitude.

A couple of striking differences from the low-density cases in Fig. 6 arise when the $N_{s p}=3$ perturbation is strengthened. First, a very clear stop band appears at $\nu_{0} \approx$ 0.75 . This instability is not observed in the low-density regime even after the $N_{s p}=3$ perturbation is increased to $1.0 \%$ of the primary focusing force. Second, several stop bands become wider. Note that the instability regions near $\nu_{0} \approx 0.75$ and 1.5 both split into two separate stop bands; the narrower but stronger stop band on the left side should be generated by a linear $(m=2)$ resonance while the other one on the right by a fourth-order $(m=4)$ resonance. In fact, the values of the coherent tune shifts are different for different modes. The coefficient $C_{m}$ in Eq. (9) gets larger for higher-order modes; according to the Sacherer's 1D theory [3], $C_{2}=0.75, C_{3}=0.88$, and $C_{4}=0.92$. This feature probably explains the split of the stop bands observed at $\nu_{0} \approx 0.75$ and 1.5. The two adjacent stop bands eventually overlap for larger perturbations, yielding one wide unstable region.

To measure the plasma-density dependence of the resonance condition, we change the number of ions initially confined in the trap. Results of three independent experiments are plotted in Fig. 9. All stop bands shift to higher values of bare tune with increasing initial ion number, as indicated by the arrows on the plot. The separation of the $m=2$ and $m=4$ stop bands becomes greater at higher density, which is believed to result from the difference between the values of $C_{2}$ and $C_{4}$.

With the present choices of parameters, some low-order nonlinear stop bands cannot be clearly identified. For

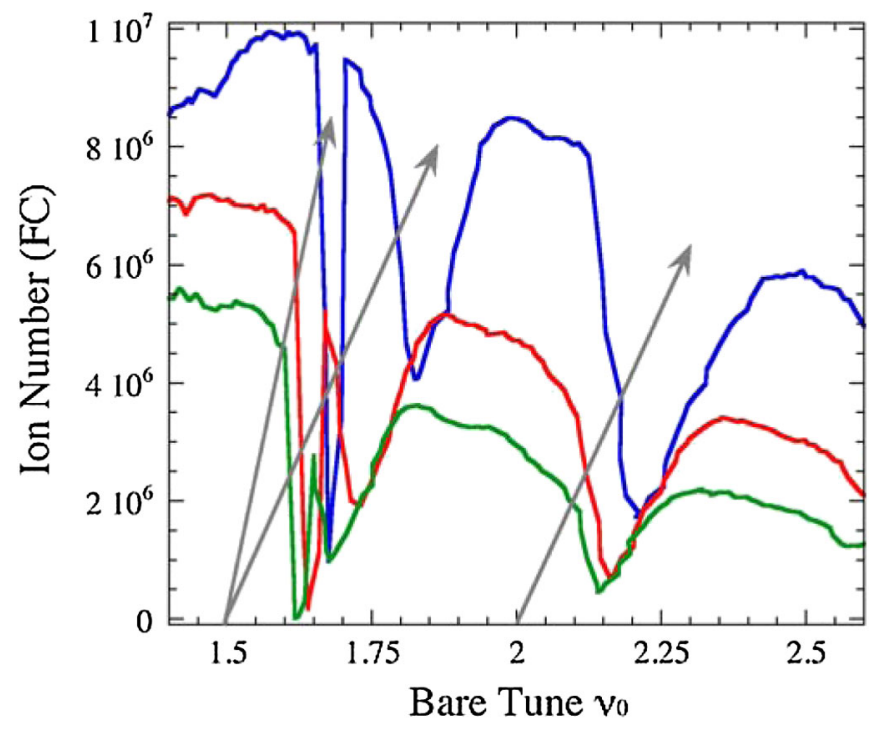

FIG. 9. (Color) Experimental results obtained under three different initial conditions (colors) of $\mathrm{Ar}^{+}$plasmas. The current of the electron beam for ionization and/or the neutral gas pressure is changed to control the initial plasma density. The amplitude of the $N_{s p}=3$ wave is $0.5 \%$ of the primary-wave amplitude. The plasma storage time is $1 \mathrm{msec}$.

example, there should be one more fourth-order stop band between $\nu_{0} \approx 0.75$ and $\nu_{0} \approx 1.5$; inserting $m=4$ and $n=3$ in Eq. (9), we have the resonance condition $\nu_{0} \approx 9 / 8$. This instability seems too weak to be resolved or perhaps is washed out due to differences between the actual experimental distribution and theoretical models. We have, however, confirmed its existence in other experiments where the amplitude of the $N_{s p}=3$ wave is made larger.

\section{Effect of field imperfections}

Any real storage ring contains weak but finite random field errors, which reduces the superperiodicity to unity. To model this situation experimentally for the case of a linear field error, we add an $N_{s p}=1$ perturbation wave as depicted in Fig. 4(c). Representative results obtained are given in Fig. 10 where two different amplitudes of the $N_{s p}=1$ wave have been considered. For reference, the $N_{s p}=3$ symmetric case is also plotted. An essential difference from the previous cases of higher lattice symmetry is found in a low-tune region where we observe the development (arrows) of two additional stop bands near $\nu_{0} \approx$ 0.5 and 1.25 . Here, instability has been enhanced by the increase of the perturbation-wave amplitude. These ion losses are likely due to linear resonance instabilities.

In order to observe the effect of the $N_{s p}=1$ wave more clearly, we extended the plasma storage time to $10 \mathrm{msec}$. As illustrated in Fig. 11, the two additional stop bands observed in Fig. 10 become more pronounced even though the amplitude of the error wave relative to the primary 


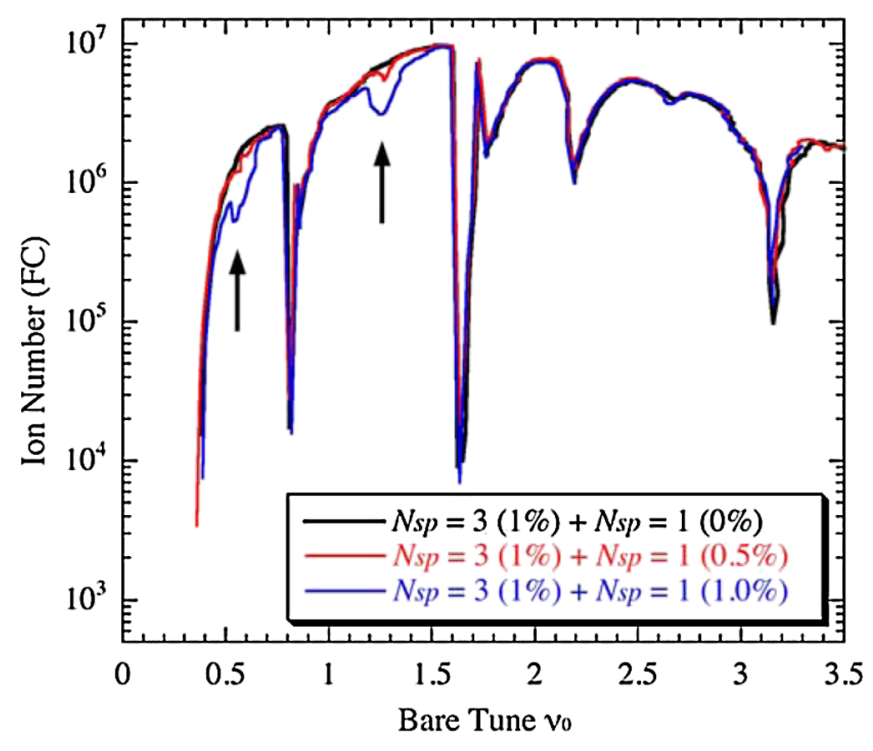

FIG. 10. (Color) Experimental results in the high-density regime where an $N_{s p}=1$ perturbation has been applied in addition to the lattice-induced wave of $N_{s p}=3$. The amplitudes of the imperfection waves $\left(N_{s p}=1\right)$ are $0 \%, 0.5 \%$, and $1 \%$ of the primary $N_{s p}=12$ focusing wave. The amplitude of the $N_{s p}=3$ wave is $1 \%$ of the primary wave in all three cases. The plasma storage time is $1 \mathrm{msec}$.

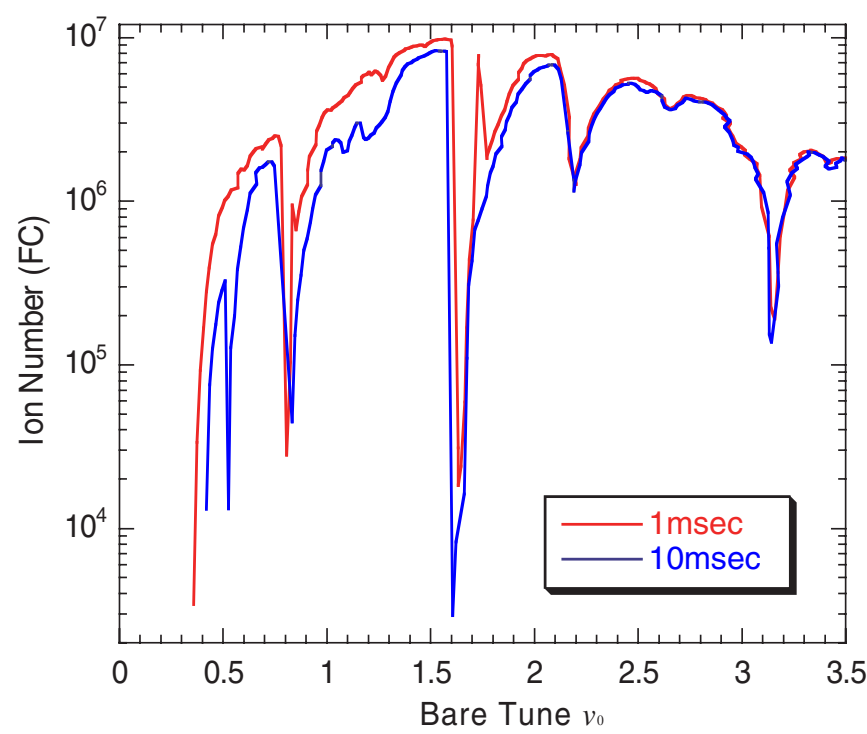

FIG. 11. (Color) Experimental results in the $N_{s p}=3(1 \%)$ plus $N_{s p}=1(0.5 \%)$ case presented in Fig. 10 contrasted for 1 and $10 \mathrm{msec}$ plasma storage times.

focusing wave is moderate. Specifically, the loss at $\nu_{0} \approx$ 0.5 has become much sharper. Another lossy region close to $\nu_{0} \approx 1$ also appears.

\section{PIC SIMULATIONS}

It is informative to compare experimental results with multiparticle numerical simulations. For this purpose, we employ the PIC code WARP $[39,40]$ that allows selfconsistent space-charge simulations including conducting electrode boundaries. Although WARP can model the full 3D geometry of the Paul trap, high resolution and particle statistics 3D simulations are time consuming and, therefore, we here apply a transverse 2D model where the plasma is axially uniform $(\partial / \partial z=0)$. Such $2 \mathrm{D}$ simulations greatly reduce computing time, thereby allowing use of high grid resolution and particle statistics. 2D simulations also give us an opportunity of checking how well SPOD reproduces the axially unbunched beam outlined in the Vlasov-Poisson model presented in Sec. II.

In the simulations, KV, waterbag, and thermal distributions are assumed initially with rms envelope radii matched to the rf focusing field. The initial waterbag and thermal distributions loaded are described in Ref. [41]. They are formed by loading a matched continuous focusing equilibrium with self-consistent space-charge-induced Debye screening effects and then canonically transforming the distributions to AG symmetry. The space-charge strength of an initial distribution is often characterized by the incoherent tune shift $\Delta \nu$, as mentioned in Sec. II. However, this parameter cannot uniquely be determined except for the KV distribution where no tune spread is present. We here compute the space-charge-depressed incoherent tune $\nu_{\text {rms }}$ by numerically integrating the rms envelope equations (without weak image contributions [42]) for matched envelope conditions. Resulting values of $\nu_{\mathrm{rms}}$ are independent of matched beam distributions (KV, waterbag, thermal). When the time structure of the rf focusing force is given, the parameters necessary for this procedure are only two, the plasma temperature and line charge density, both of which are set in WARP simulations. The rms tune depression is defined by $\eta=\nu_{\text {rms }} / \nu_{0}$, which also allows us to evaluate the incoherent tune shift $\Delta \nu=$ $\nu_{0}-\nu_{\mathrm{rms}}=(1-\eta) \nu_{0}$. Assuming that the plasma is in thermal equilibrium, the transverse temperature should be on the order of $0.1 \mathrm{eV}$ according to past experiments [9]. In the present simulation study, we typically fix the plasma temperature in the range $0.1-0.2 \mathrm{eV}$ and then adjust the rms tune depression by changing the line charge density.

Figure 12 shows the ratio of the rms emittance to its initial value after $0.1 \mathrm{msec}$ when the initial ion distribution is the waterbag type. Initial $\mathrm{KV}$ and thermal distributions obtain qualitatively similar results [43]. In order to enhance nonlinear resonances, we artificially introduced the thirdand fourth-order multipole rf components that model electrode misalignments. The amplitudes of these low-order nonlinearities are chosen, respectively, as $6 \%$ and $1 \%$ of the quadrupole amplitude, which is probably much greater than the actual error fields. Then, the 100 primary rf periods that the simulations are advanced are enough to identify the locations of low-order stop bands because of the rapid plasma breakup on resonance as experimentally confirmed in Fig. 8. In Fig. 12(a) where the rf perturbation 

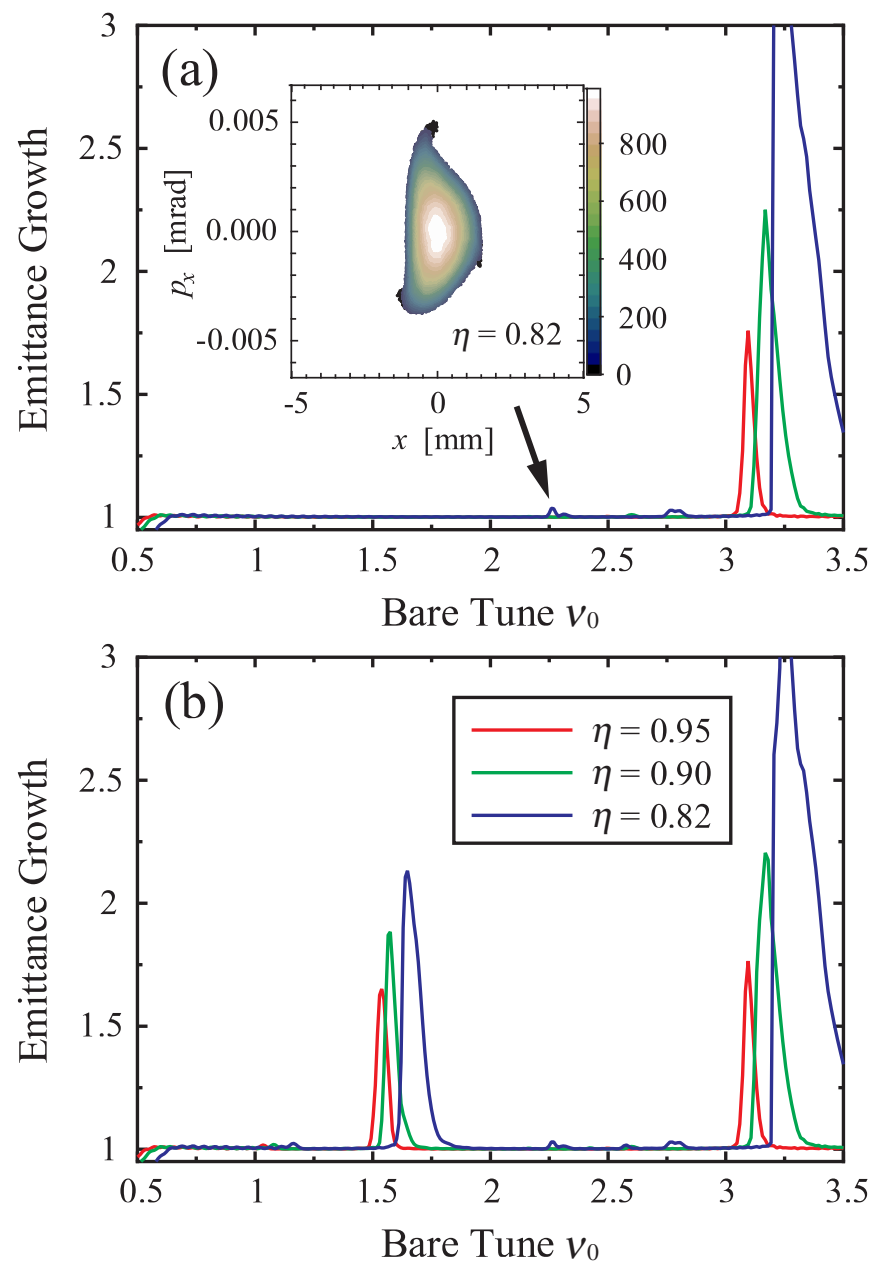

FIG. 12. (Color) PIC simulation results showing emittance growth after $0.1 \mathrm{msec}$ as a function of the bare tune $\nu_{0}$. The initial distribution is waterbag form $\mathrm{rms}$ matched to the $\mathrm{rf}$ focusing force. Three different tune depressions $\eta$ are shown to confirm the stop band shifts. The upper and lower figures correspond to the following cases: (a) $N_{s p}=12$ (no perturbation wave applied), and (b) $N_{s p}=3$ (the lattice-induced wave added, whose amplitude is $0.5 \%$ of the primary focusing-wave amplitude). In (a), the transverse phase-space projection of the plasma observed after $100 \mathrm{rf}$ periods is shown for $\nu_{0} \approx 2.25$.

is absent $\left(N_{s p}=12\right)$, no severe resonances are observed below $\nu_{0}=3$ [44]. A weak stop band, however, exists in the region near $\nu_{0} \approx 2.25$ and $\nu_{0} \approx 2.75$, which agrees with the experimental observations (see Fig. 7). According to the coherent resonance theory, the former stop band is third order and must be there even without the perturbation rf wave. The distribution distortions in the phase-space plot in Fig. 12(a) suggest that the emittance growth is actually caused by third-order instability. In Fig. 12(b), an $N_{s p}=3$ perturbation wave is superimposed on the primary focusing field. Another strong instability has appeared near $\nu_{0} \approx$ 1.5. Although no rapid emittance growth is observed around $\nu_{0} \approx 0.75$ unlike the experimental result in Fig. 7, we verified the existence of the stop band near $\nu_{0} \approx$
0.8 in separate simulations with increased perturbationwave amplitude. Careful inspection of the numerical data also revealed that the peak of $\nu_{0} \approx 1.5$ and even the tiny third-order peak of $\nu_{0} \approx 2.25$ are formed by not a single but at least two separate resonances overlapping each other.

Since the instability regions experimentally observed are well explained by PIC simulations, we can make a fairly accurate estimate of effective tune depression by comparing the numerical results and experimental data. (We are now preparing laser-induced fluorescence diagnostics which allow direct measurement of transverse plasma temperature of ${ }^{40} \mathrm{Ca}^{+}$plasmas.) As briefly described in Sec. II, it is interesting and practically important to ask whether the shift of an experimentally observed stop band as a whole is smaller or larger than the incoherent tune shift $\Delta \nu$. Figure 13 addresses this question using the experimental data in Fig, 9 and corresponding WARP simulations. The ordinate represents the location of a particular resonance stop band measured from its position at zero space charge. For example, in Fig. 9, the $m=2$ stop band approaches the $\nu_{0}=1.5$ line (the origin of the left end arrow) as we reduce the plasma density. We thus subtract 1.5 from the bare-tune values where particle losses are observed due to the $m=2$ resonance, and plot them in Fig. 13. The vertical bar corresponds to the FWHM (full width at half maximum)

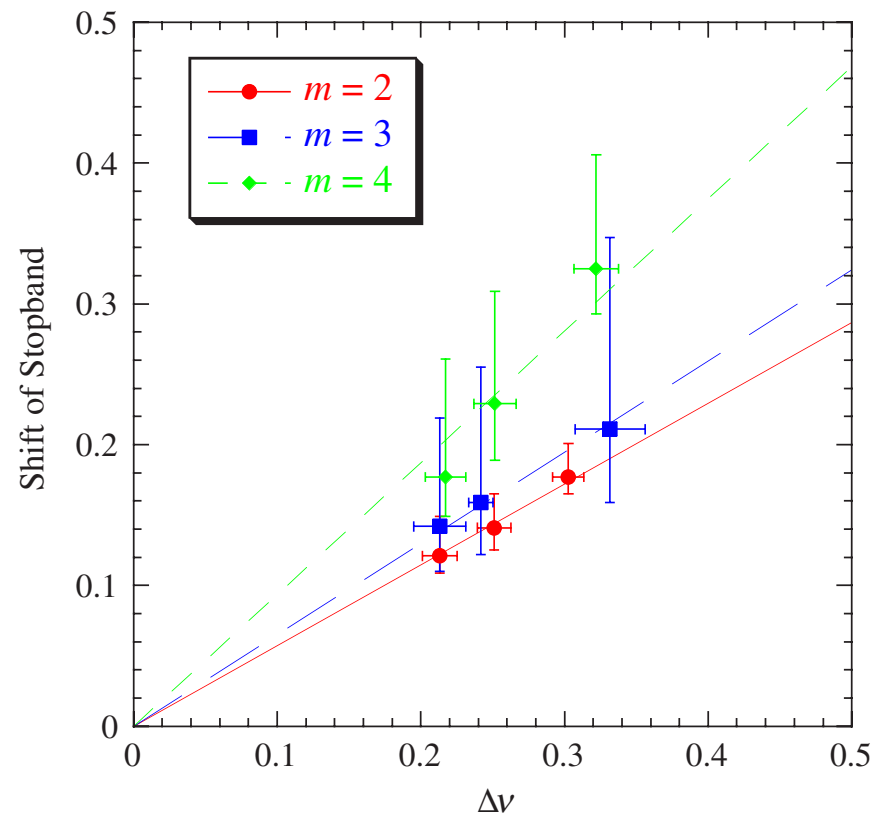

FIG. 13. (Color) Resonance stop bands' shifts versus rms incoherent tune shift. The experimentally observed shifts and widths of the three stop bands in Fig. 9 are plotted as a function of the rms incoherent tune shift $\Delta \nu$. The tune depression $\eta$ is estimated by comparing PIC simulation results and experimental data. The vertical bars represent the FWHM of the stop bands. Circles (0), squares $(\boldsymbol{\square})$, and diamonds $(\bullet)$ indicate the locations where the maximum ion loss occurs within the $m=2, m=3$, and $m=4$ stop bands in Fig. 9. 
of the stop band. The abscissa is $\Delta \nu$ estimated from the experiments and simulations. Circles $(\bullet)$ and diamonds $(\diamond)$ are the distances in $\nu_{0}$ from the $\nu_{0}=1.5$ line to the $m=2$ and $m=4$ stop bands, respectively. Squares ( $\square$ ) show the distance of the $m=3$ stop band measured from the $\nu_{0}=2$ line. Figure 13 suggests that the average shifts of the three stop bands are less than (and roughly proportional to) the incoherent detuning $\Delta \nu$; in other words, the constant factor $C_{m}$ in the resonance condition (9) appears to be less than unity. A tendency that a higher-order stop band shifts more at a particular plasma density is also evident from Fig. 13. The detunings evaluated at the right edges of the wide nonlinear ( $m=3$ and $m=4)$ stop bands in Fig. 9, however, are close to $\Delta \nu$. According to the resonance condition in Eq. (9), these instability regions potentially contain several resonances of different orders ( $m$ numbers) with different $n$ numbers. The experimentally observed stop bands might thus be made somewhat wider, due to resonance overlapping, than the actual widths of the $m=2,3$, and 4 stop bands.

\section{CONCLUDING REMARKS}

A systematic study of coherent betatron resonances was performed with the new experimental tool "S-POD" that approximately reproduces the center-of-mass frame dynamic motion of a charged-particle beam evolving in a periodic focusing lattice. S-POD enables us to explore a wide range of system parameters, which is often difficult to accomplish in conventional experiments relying on largescale accelerators. $\mathrm{Ar}^{+}$plasmas of various densities were generated and confined in a compact linear Paul trap for novel resonance experiments. In order to examine how the resonance conditions are influenced by lattice properties, we perturbed the plasma with superimposed rf waves that represent the effect of lattice superperiod and imperfection fields of a particular storage ring (see Fig. 4). We observed considerable loss of ions when the coherent resonance condition in Eq. (9) is satisfied. The strengths of those stop bands depend on the lattice; $m$ th-order resonances are not always equally severe for different $n$ values in Eq. (9). In the present examples where a simple threefold symmetric lattice is analyzed, strong resonances took place at bare betatron tunes slightly above $0.75,1.5,2$, and 3 (see, e.g., Fig. 7). The experimental data clearly indicate that the stop bands near $\nu_{0} \approx 0.75$ and 1.5 consist of two separate instabilities for which second- and fourth-order resonances are probably responsible. The other two stop bands near $\nu_{0} \approx 2$ and 3 appear mainly due to third- and second-order resonances. We confirmed slight shifts of these stop bands induced by the space-charge force which depresses the effective tune. Consistently, as illustrated in Fig. 9, all stop bands shift toward higher bare-tune values with increasing plasma density. We also verified the effect of imperfect lattice fields by superimposing another perturbation wave. It is evident from Figs. 11 and 12 that the error wave produces additional weak stop bands. These experimental observations are in good qualitative agreement with 2D PIC simulations.

The novel experimental system described here is a useful tool to explore a diverse range of beam physics. Experiments with S-POD are particularly powerful in investigating self-field-induced collective phenomena whose systematic studies are difficult to perform both computationally and experimentally. The three curves in Fig. 7 are composed of experimental data obtained from nearly 1000 independent measurements; nevertheless, it took us only a few hours to accumulate the data. The S-POD control system automatically executes necessary experimental procedures without human intervention to retune parameters. If such a large number of detailed 3D numerical space-charge simulations are carried out, the run time could be prohibitive even with high-performance parallel computers. In our present system, the most timeconsuming part of the experimental procedure is the data transfer from the detector to the computer, which takes some seconds. This means that the total time needed for the 1000 measurements is almost unchanged even if we extend the plasma storage period by a factor of 10 . In contrast, the required CPU time for numerical simulations becomes 10 times longer. Note further that in accelerator experiments, it is not acceptable to have significant particle losses from a high-power beam because it may damage the machine. Severe restrictions are therefore often imposed on accelerator-based studies of space-charge effects. Although the S-POD experiments also have limitations, such a new approach provides an alternative way to deepen understanding of collective beam effects.

In future applications of S-POD, we anticipate developing improved plasma diagnostics to allow more detailed exploration of beam physics phenomena. Full phase-space diagnostics will be developed. The small physical scale of the system and the resulting economy of lattice reconfigurations together with good shot-to-shot reproducibility and low particle energy enable a wide range of diagnostic options to be considered. Flexibility in ion species stored enables us application of laser diagnostics for nondestructive, time-resolved phase-space measurements. Ongoing experiments on Coulomb crystals in another Paul trap employ lasers which can be adapted for this purpose [45].

\section{ACKNOWLEDGMENTS}

The authors wish to thank A. M. Sessler for his valuable comments on this paper. We are indebted to D. P. Grote and J.-L. Vay for support provided on the WARP simulation code. This work was supported in part by a Grant-in-Aid for Scientific Research, Japan Society for the Promotion of Science, by the High Energy Accelerator Research Organization, and by Lawrence Livermore National Laboratory under U.S. Department of Energy Contract No. DE-AC52-07NA27344. 
[1] M. Reiser, Theory and Design of Charged Particle Beams (Wiley, New York, 1994).

[2] J.D. Lawson, The Physics of Charged-Particle Beams (Oxford University Press, Oxford, 1988) 2nd ed.

[3] F.J. Sacherer, Ph.D thesis, Lawrence Radiation Laboratory [Report No. UCRL-18454, 1968].

[4] See, e.g., Proceedings of the 39th ICFA Advanced Beam Dynamics Workshop on High Intensity High Brightness Hadron Beams (KEK, Tsukuba, Japan, 2006).

[5] S. Y. Lee, Space Charge Dominated Beams and Applications of High Brightness Beams, AIP Conf. Proc. No. 377 (AIP, New York, 1995).

[6] See, e.g., Proceedings of the Workshop on Beam Cooling and Related Topics (GSI, Bad Kreuznach, Germany, 2007).

[7] D. M. Maletic and A. G. Ruggiero, Crystalline Beams and Related Issues (World Scientific, Singapore, 1996).

[8] H. Okamoto, Y. Wada, and R. Takai, Nucl. Instrum. Methods Phys. Res., Sect. A 485, 244 (2002).

[9] R. Takai, H. Enokizono, K. Ito, Y. Mizuno, K. Okabe, and H. Okamoto, Jpn. J. Appl. Phys. 45, 5332 (2006).

[10] W. Paul and H. Steinwedel, Z. Naturforsch. A 8, 448 (1953).

[11] P. K. Ghosh, Ion Traps (Oxford Science, Oxford, 1995), and references therein.

[12] D. J. Berkeland, J. D. Miller, J. C. Bergquist, W. M. Itano, and D. J. Wineland, Phys. Rev. Lett. 80, 2089 (1998).

[13] M. Drewsen, C. Brodersen, L. Hornekær, J. S. Hangst, and J. P. Schiffer, Phys. Rev. Lett. 81, 2878 (1998).

[14] N. Kjærgaard, K. Molhave, and M. Drewsen, Phys. Rev. E 66, 015401(R) (2002).

[15] J. I. Cirac and P. Zoller, Phys. Rev. Lett. 74, 4091 (1995).

[16] T. Baba and I. Waki, Jpn. J. Appl. Phys. 35, L1134 (1996).

[17] K. Matsubara, U. Tanaka, H. Imajo, S. Urabe, and M. Watanabe, Appl. Phys. B 76, 209 (2003).

[18] R. C. Davidson, H. Qin, and G. Shvets, Phys. Plasmas 7, 1020 (2000).

[19] E.P. Gilson, R.C. Davidson, P.C. Efthimion, and R. Majeski, Phys. Rev. Lett. 92, 155002 (2004).

[20] E. P. Gilson, M. Chung, R. C. Davidson, P. C. Efthimion, and R. Majeski, Phys. Rev. ST Accel. Beams 10, 124201 (2007).

[21] M. Chung, E. P. Gilson, M. Dorf, R. C. Davidson, P. C. Efthimion, and R. Majeski, Phys. Rev. ST Accel. Beams 10, 014202 (2007).

[22] H. Okamoto and H. Tanaka, Nucl. Instrum. Methods Phys. Res., Sect. A 437, 178 (1999).

[23] I. Hofmann, L. J. Laslett, L. Smith, and I. Haber, Part. Accel. 13, 145 (1983).

[24] H. Okamoto and K. Yokoya, Nucl. Instrum. Methods Phys. Res., Sect. A 482, 51 (2002).

[25] In many previous analytic theories of collective resonances, the lattice periodicity has been smoothed out for the sake of mathematical simplicity, which eliminates the instabilities studied here. A periodic perturbation is, therefore, introduced later to excite resonances.

[26] E. D. Courant and H. S. Snyder, Ann. Phys. (Leipzig) 3, 1 (1958).

[27] H. Wiedemann, Particle Accelerator Physics (Springer, New York, 2007), 3rd ed.

[28] R. L. Gluckstern, in Proceedings of the Linear Accelerator
Conference (Fermilab, Batavia, USA, 1970), p. 811.

[29] A. V. Fedotov, I. Hofmann, R. L. Gluckstern, and H. Okamoto, Phys. Rev. ST Accel. Beams 6, 094201 (2003).

[30] R. Baartman, in Proceedings of the Workshop on Space Charge Physics in High Intensity Hadron Rings (AIP, Shelter Island, 1998), p. 56.

[31] D. R. Denison, J. Vac. Sci. Technol. 8, 266 (1971).

[32] R. Takai, K. Nakayama, W. Saiki, K. Ito, and H. Okamoto, J. Phys. Soc. Jpn. 76, 014802 (2007).

[33] For more information, see http://www.aetjapan.com/ english/software/index.html.

[34] LABVIEW is a graphical programming tool developed by National Instruments; for more information, see http:// www.ni.com/labview.

[35] Note, however, that all physical information necessary to describe the resonant instability is contained in a single lattice period; we do not have to take a look at the whole ring if it holds perfect $N_{s p}$-fold symmetry.

[36] Provided that the superperiodicity is an odd number, strong intrinsic linear resonances can be encountered at quarter integers as predicted by Eq. (9).

[37] This situation is similar to what is expected in highintensity hadron machines. For example, the bunch length of a proton beam in the J-PARC $3 \mathrm{GeV}$ ring is about $90 \mathrm{~m}$ at injection and has a transverse extent less than $0.1 \mathrm{~m}$, which means that the beam is like a very long cylinder rather than an ellipsoid. A beam confined in a barrier bucket should also be similar to the present case. In SPOD, a long cylinderlike plasma can be produced by elongating the quadrupole electrodes.

[38] In the case where $N_{s p}=12$, the coherent resonance condition reads $\nu_{0}-C_{m} \Delta \nu \approx 6 n / m$. Therefore, a linear stop band $(m=2)$ appears near $\nu_{0} \approx 3$ and a third-order stop band near $\nu_{0} \approx 2$.

[39] D. P. Grote, Ph. D thesis, University of California at Davis, 1994.

[40] D. P. Grote, A. Friedman, G. D. Craig, I. Haber, and W. M. Sharp, Nucl. Instrum. Methods Phys. Res., Sect. A 464, 563 (2001).

[41] S. M. Lund, T. Kikuchi, and R. C. Davidson, Phys. Rev. ST Accel. Beams 12, 114801 (2009).

[42] To make a quick estimate of the image effect, consider a long plasma column surrounded by a cylindrical pipe. When the plasma extent is one-third of the pipe radius, the image-induced tune shift is only $2 \%$ of the image-free tune for a plasma that has a tune depression of $\eta=0.8$ [1]. The transverse size of an ion plasma in our Paul trap is typically less than one-third of the distance from the axis to the electrode surface.

[43] The locations of stop bands are almost independent of the initial distributions though the stop band widths are somewhat different; the stop bands of an initial Gaussian plasma tend to be widest and those of an initial KV plasma narrower than the other two.

[44] The particle loss in the low-tune region $\left(\nu_{0}<0.6\right)$ is not related to instability, but rather is the result of weak focusing forces leading to increased matched plasma size and scraping of the plasma by the quadrupole electrodes.

[45] K. Izawa, K. Ito, H. Higaki, and H. Okamoto (unpublished). 